\title{
La investigación como estrategia para el mejoramiento de la convivencia escolar y la construcción de ciudadanía*
}

\author{
Andrés David Medina Palacios* \\ Gabriel Enán Moreno Mosquera** \\ Jackeline Rosero López \\ Raúl García Mosquera ${ }^{* * * *}$
}

Recibido: 10 de julio de 2016

Enviado a pares evaluadores: 18 de julio de 2016

Aprobado por pares evaluadores: 14 de septiembre de 2016

Aprobado por Comité Editorial: 4 de octubre de 2016

DOI: 10.22395/csye.v5n10a4

\section{RESUMEN}

Este artículo recoge algunos de los hallazgos de la investigación "Incidencia de los comités escolares de convivencia en la formación de habilidades democráticas y ciudadanas, el caso de la Institución Educativa Pedro Grau y Arola de la ciudad de Quibdó" (2016), en la que, a partir de recolección de información sobre convivencia en fuentes bibliográficas y con la comunidad educativa focalizada, se construye una propuesta donde es posible transversalizar la investigación en el aula y la formación ciudadana de los estudiantes.

El trabajo investigativo, de corte cualitativo, se desarrolló con estudiantes de los grados $4 .^{\circ} \mathrm{y}$
$9 .^{\circ}$, de edades entre los 10 y 16 años, a partir de encuestas, entrevistas y desarrollo de grupos focales.

Finalmente el trabajo concibe la investigación escolar como una pertinente y muy necesaria estrategia que permite a los estudiantes identificar los contextos donde se desenvuelven, al tiempo que desarrollan habilidades para desempeñarse en ellos de manera respetuosa, dando pie, así, al componente de la formación democrática y ciudadana.

Palabras clave: convivencia escolar; conflicto; participación; democracia; ciudadanía.

\footnotetext{
Resultado de la investigación "Incidencia de los comités escolares de convivencia en la formación de habilidades democráticas y ciudadanas, el caso de la Institución Educativa Pedro Grau y Arola de la ciudad de Quibdó", presentada a la Maestría en Conflicto y Paz de la Universidad de Medellín en 2016.

** Licenciado en Ciencias Sociales de la Universidad Tecnológica del Chocó "Diego Luis Córdoba". Magíster en Conflicto y Paz de la Universidad de Medellín. En la actualidad labora como funcionario UTL en el Senado de la República de Colombia. Correo electrónico: andamepa84@hotmail.com

*** Licenciado en Ciencias de la Educación, con especialidad en Idiomas, de la Universidad Tecnológica del Chocó "Diego Luis Córdoba". Especialista en Lengua Materna (Español). Abogado de la misma universidad. Especialista en Derecho Administrativo de la Universidad Nacional de Colombia. Magíster en Conflicto y Paz de la Universidad de Medellín. En la actualidad labora como docente adscrito a la Facultad de Educación en el Programa de la Licenciatura en Lingüística y Literatura de la Universidad "Diego Luis Córdoba". Correo electrónico: gabrielen10@hotmail.com

**** Psicóloga y especialista en Salud Ocupacional y Riesgos Psicosociales de la Universidad Santiago de Cali. Magíster en Conflicto y Paz de la Universidad de Medellín. Labora en la actualidad en la Escuela Superior de Administración Pública. Correo electrónico: jroserolop@gmail.com

***** Licenciado en Matemáticas y Física de la Universidad Tecnológica del Chocó Diego Luis Córdoba. Especialista en Gestión Educativa de la Fundación Universitaria Luis Amigó. Magíster en Conflicto y Paz de la Universidad de Medellín. Labora actualmente como profesional especializado en la Universidad Tecnológica del Chocó "Diego Luis Córdoba". Correo electrónico: raulgarciamosquera@gmail.com
} 


\section{Research as a strategy for improving school life and the construction of citizenship}

\section{ABSTRACT}

Article summarizes the main findings and 9 , aged between 10 and 16 years from of the investigation "Incidence of school committees coexistence in the formation of democratic and citizenship skills, the case of School Pedro Grau y Arola city of Quibdó" (2016), developed by students MA in Conflict and Peace at the Universidad de Medellín, which from gathering information on coexistence in literature sources and the targeted educational community, a proposal where it is possible to mainstream classroom research and citizen education is built of the students.

The investigative work, qualitative, developed with students from grades 4 surveys, interviews and development of group work.

Finally the work conceived scholarly research as a relevant and much needed strategy that allows students to identify the contexts in which they operate, while developing skills to perform them in a respectful manner, thus giving rise to the component of democratic and civic education.

Keywords: School Coexistence; conflict; participation; democracy; citizenship. 
El maestro no es el que sabe, es decir, el que posee el conocimiento y lo transmite, sino el que sabe dónde está el conocimiento y conoce los procedimientos que garantizan el aprendizaje más eficaz

EMILIO TENTI FANFANI

\section{Presentación}

Trabajar sobre estrategias de indagación como alternativa para el mejoramiento de las relaciones entre los estudiantes, de tal manera que faciliten la reflexión y el compromiso con la formación democrática y ciudadana es una oportunidad para una escuela que en ocasiones se siente cansada, carente de discurso y en crisis, donde se evidencia de manera nítida la precariedad de los vínculos humanos, que como lo propone el filósofo polaco Zygmunt Bauman, la liquidez pareciera ser la medida de todas las cosas, pues esta terminó por ser una sociedad individualista y privatizada, marcada por el carácter transitorio y volátil de sus relaciones; por ello, afirma Bauman (Citado por Vásquez, 2008):

El amor se hace flotante, sin responsabilidad hacia el otro, se reduce al vínculo sin rostro que ofrece la web. Surfeamos en las olas de una sociedad líquida siempre cambiante -incierta- y cada vez más imprevisible, es la decadencia el Estado del bienestar. La modernidad liquida en un tiempo sin certezas, donde los hombres que lucharon durante la ilustración por obtener libertades civiles de deshacerse de la tradición, se encuentran ahora con la obligación de ser libres asumiendo los miedos y angustias existenciales que tal libertad comporta (p. 1).

Desde el punto de vista metodológico, el desarrollo de la investigación se respaldó en el enfoque cualitativo, aunque se utilizaron algunos datos estadísticos. Se empleó el método investigación-acción, para lo cual se aplicaron 70 encuestas a estudiantes de los grados $4 .^{\circ}$ y $9 .^{\circ}$, con edades entre los $10 \mathrm{y}$ 16 años. Seguidamente se aplicaron entrevistas a 6 directivas y docentes y una guía de observación que permitiera reconocer el contexto institucional y del territorio.

Respecto al contexto es pertinente destacar que la Institución Educativa Pedro Grau y Arola se encuentra ubicada en la zona norte de Quibdó, catalogada como una de las más vulnerables de la ciudad, donde, además de todos los desajustes sociales relacionados con la pobreza extrema, hacen presencia grupos al margen de la ley. Esa es, además, una de las zonas que concentra mayor población víctima de la violencia, como bien lo demuestra el gráfico número 1. 


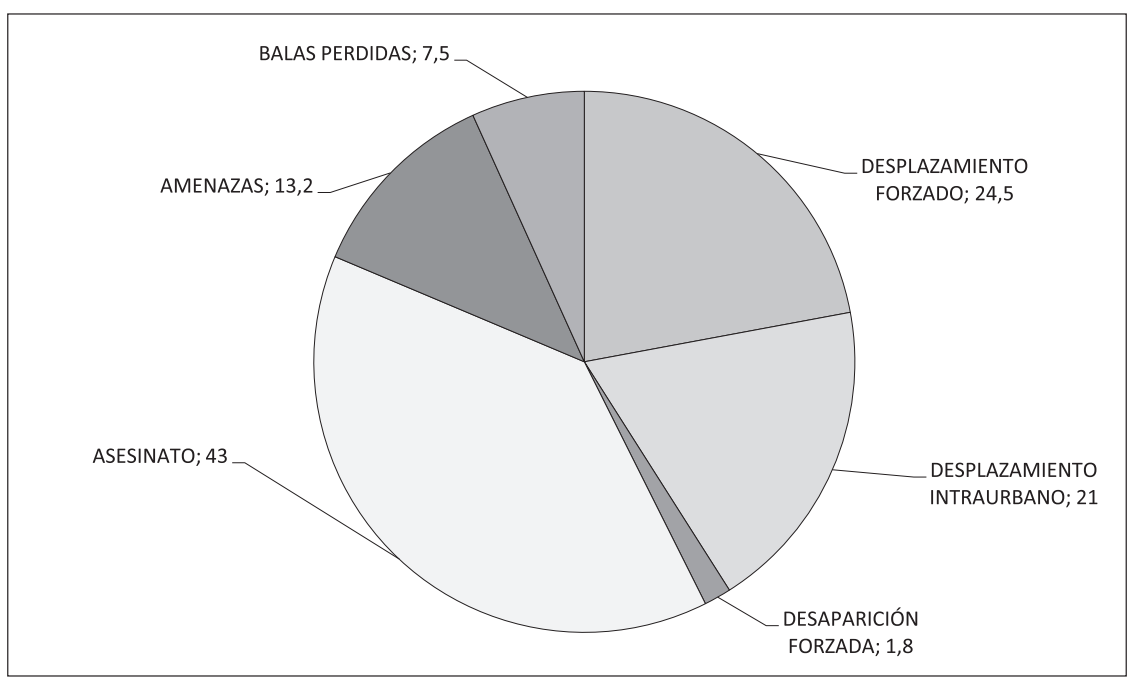

Gráfico $\mathbf{N} .^{\circ}$ 1: Tipos de hechos victimizantes que han padecido las familias de los estudiantes Fuente: Gráfico construido por los autores a partir de la aplicación de encuesta

Esta zona de la ciudad es habitada por personas en extrema pobreza, cuyas familias se ocupan generalmente en labores informales. En estos barrios existe una fuerte presencia de grupos armados ilegales que controlan la vida pública y privada de las comunidades, lo que hace de los estudiantes un grupo vulnerable, ya sea por resultar afectados por las acciones de estos grupos o por ser utilizados por ellos; por esto el reclutamiento forzado es una de las preocupaciones más sentidas por esta comunidad.

Cabe resaltar que esta zona de la ciudad también posee importantes organizaciones sociales y comunitarias que promueven la cultura, la participación y la autogestión; de allí que haya posibilidades para la institución de fortalecer la convivencia, la cooperación y el ejercicio de la ciudadanía, de tal manera que se sensibilice a los estudiantes sobre las problemáticas de sus entornos, al tiempo que, a través de los acercamientos investigativos, se comprometen ética, políticamente y moralmente con ellos.

\section{Antecedentes}

La investigación en el campo educativo fue una labor esencialmente concentrada en las instituciones de educación superior y en sus docentes, pues durante décadas se consideró que las instituciones de educación básica y media solo reproducían saberes que el mundo "científico" y la academia generaban, desconociéndose tanto los procesos investigativos que podían darse en estas instituciones como los aportes que al respecto pudieran hacer sus maestros (MEN, 2002). 
Tal vez con el denominado mundo informacional (Castells, 2002) es como la generación de conocimiento ha tomado un importante lugar en las instituciones de la educación, incluida la básica, al generar toda una corriente de pensamiento que reconoce otros lugares y formas de producir conocimiento asumidos como válido. Es así como las ONG, las organizaciones comunitarias, las instituciones de educación básica, entre otras, van a tomar importancia en lo que respecta a la generación de saberes desde sus aulas, hasta el punto que investigadoras como la profesora Marina Camargo, de la Universidad de la Sábana se pregunta: "Maestro investigador, ¿y por qué no?" (2005, p. 24).

Pero esta es una corriente que empieza en el país a principios del siglo XX y que va a desencadenar literatura, organización y eventos en los niveles local, nacional e internacional, que inciden en los procesos investigativos generados desde las aulas escolares; de ello dan cuenta los procesos acompañados por el Programa Ondas, de Colciencias, dedicado a promover y financiar proyectos investigativos escolares en las diferentes regiones del país. En esta misma dirección Maloka en Bogotá y el Parque Explora en Medellín vienen promoviendo diversas ferias donde la tarea esencial es la difusión de los trabajos investigativos que vienen adelantando las escuelas y colegios del país.

Estos logros, de por sí loables, han sido acompañados de una visión fundamental, esto es, el asumir la formación en el campo de la investigación como una tarea que debe estar acompañada de la construcción de ciudadanía (ColcienciasOndas, 2007), pues de poco serviría formar más y mejor a unos sujetos, si estos no se perfilan como respetuosos de los derechos de sus semejantes.

No se trata, pues, de utilizar diversas metodologías para acercarse a un conocimiento más elaborado; se trata, de manera fundamental, de la formación de una cultura política democrática a partir del desarrollo de competencias investigativas; se trata de cómo comprender los usos políticos de la práctica de la investigación desde la escuela, lo que exige reconocer que educar en este campo involucra diferentes ámbitos como son la dimensión afectiva, la intelectual, la cognoscitiva, la ética y la convivencial.

La apuesta debe ser la de formar integralmente ciudadanos líderes, responsables de sus decisiones individuales y colectivas, críticos y reflexivos frente a la realidad y al acontecer social (Ospina, 2015). Hombres y mujeres con voluntad para aprender y desaprender, capaces de emprender acciones para vivir con otros en medio de la diferencia.

\section{Educación y ciudadanía}

Educar en y para el ejercicio de la ciudadanía es una de las tareas fundamentales del sistema educativo; al contrario, en Colombia el desarrollo de propuestas en 
materia de derechos en la mayoría de las instituciones se ha inclinado más por el enfoque normativista que por la perspectiva de promoción de la convivencia escolar, de la ciudadanía y de los derechos humanos, lo que no deja espacios para la construcción de escenarios propicios para la formación ciudadana (Agudelo, 2006).

Sociedades como la colombiana, que han experimentado sucesivas oleadas de violencia, requieren con urgencia de procesos en los que se procure la formación para el ejercicio de la ciudadanía desde la perspectiva de los derechos humanos, todo ello enmarcado en la trasformación de prácticas cotidianas que permitan generar una cultura de paz (García y Ospina, 2015).

Lo anterior es bien interesante, pues la primera vez que los seres humanos se reúnen para darse un proyecto de humanidad es cuando proclaman la Declaración Universal de Derechos Humanos, que al fin de cuentas es el principal proyecto de humanidad de que hoy se dispone; por eso los derechos humanos no pueden ser un tema, una unidad o una dimensión a trabajar en las escuelas y colegios del país; estos deben ser el norte ético del proyecto democrático. De allí se concluye que solo es posible construir la democracia en una sociedad que se cimienta sobre la lógica de los derechos humanos y, más aún, sobre la cultura de los derechos, que engloba el respeto que es necesario tener también por todos aquellos seres no humanos (los animales, el medio ambiente), que también merecen aceptación, consideración y protección (Gil, 2011).

Pero el problema de la ciudadanía y de la democracia no se resuelve imponiendo en las instituciones cursos o temas relacionados con la cultura de los derechos y la democracia; son necesarias transformaciones sociales y culturales de la sociedad toda.

Los cambios que se necesitan son de fondo, y deben ir más allá de las personas, pues tienen que ver con las instituciones, con la creación de prácticas y hábitos democráticos y con la recuperación de la libertad democrática (Maguendzo, 2003). No se trata de pensar que la escuela como institución social vaya a producir los grandes cambios que la cultura política de los colombianos necesita, pero sin educación ningún cambio será efectivo; por ello los compromisos que asume una sociedad, además de éticos, deben ser morales, entendiendo que la moral, en ocasiones, puede ser más importante que las mismas leyes para alcanzar una sociedad más tolerante y pacífica, pues si como se dice una imagen vale más que mil palabras, podría decirse que una convicción moral vale más que mil leyes.

En este orden de ideas se hace necesario entender la democracia más que como algo instrumental, como un estilo de vida. Es la concepción de la ciudada- 
nía activa; es la idea de la democracia no como algo que se ve, se hace o de lo que se habla, en no pocas ocasiones con escasa información. Es la noción de la democracia como el espacio de la acción política, entendida como el uso efectivo de los derechos y capacidad de actuar con otros de manera cooperativa para transformar los problemas de los entornos más inmediatos de los sujetos. Así planteadas las cosas, es evidente que la ciudadanía no es dada, no es otorgada, es una construcción colectiva (Ospina, 2015).

Un ciudadano es la persona que es capaz, en cooperación con otros, de construir o transformar las leyes y normas que él mismo quiere vivir, cumplir y proteger para la dignidad de todos; en este sentido es posible afirmar que una escuela democrática es una escuela que forma ciudadanos. Pensar una escuela y una pedagogía para formar ciudadanos es muy distinto que pensarla para hacer buenos estudiantes (Jares, 1997). En una educación democrática el problema es cómo transformar cosas que se tienen para transformar para formar ciudadanos, pues desde el punto de vista de la democracia, las escuelas no tienen estudiantes, lo que tienen son personas que se van transformando en ciudadanos.

Es desde la anterior concepción que surge la noción de la escuela como el primer espacio de actuación pública del niño, siendo su primer escenario de contacto con lo público, con lo común y lo colectivo; de allí que sea un error hablar de la escuela como el segundo hogar, pues entre más parecida sea una institución educativa a un hogar, menos democrática será.

Un ciudadano tiene que ser capaz de leer su contexto si realmente se asume como sujeto de derechos y de deberes. Esto es fundamental porque los medios informativos por excelencia de los estudiantes, y de buena parte de la ciudadanía, son los medios masivos, con toda su carga de intereses ideológicos, políticos y económicos, lo que no da pie a concebir una información y una formación realmente diversa; por lo mismo, es necesario ubicarse de manera reflexiva y crítica frente a las relaciones de dominación y exclusión que estos imponen.

Si se parte del principio de que una opinión política es una opinión cualificada, se concluye necesariamente que el ciudadano necesita conocer para opinar; de allí la exigencia de dotar a los estudiantes de herramientas para que puedan comprender y participar en los asuntos públicos. Al fin de cuenta, asumir que lo pedagógico lleva implícito el saber, la disciplina, el discurso y la práctica.

En este sentido los procesos educativos en el campo de la democracia y la ciudadanía deben poner en juego los saberes propios, las lecturas del contexto, el debate argumentado y la construcción de propuestas de investigación y de intervención; es necesario igualmente entender que el orden social es una 
construcción humana y como tal se puede transformar, lo que exige, además, entender que una sociedad debe estructurarse mediante un proceso de inclusión y no de exclusión, para representar y reconocer a todos sus miembros.

Es necesario promover desde las aulas escolares la potenciación de escenarios de inclusión desde un enfoque diferencial, es decir, marcos de comprensión y dispositivos que combatan la discriminación por razón de identidad cultural o étnica, sexo, orientación sexual, religión u origen, entre otros, con el fin de generar relaciones y condiciones de equidad, lo cual bien se puede lograr a través de la implementación de procesos investigativos en las aulas.

Es necesaria la formación de sujetos capaces de desarrollar una actitud ética frente a la vida, que reclama la capacidad para comprender que las acciones y decisiones particulares inciden en las demás vidas y en el entorno; es decir, tienen consecuencias, en una palabra, sujetos capaces de ejercer la ciudadanía.

Felisa Tibbitts sugiere que para ejercer la ciudadanía de manera activa es necesario "desarrollar un sentido de identidad local y global, además de la habilidad de comprender a los demás y apreciar la diversidad" (Tibbitts, 2007, p. 75), lo que sugiere el trabajo denodado en aulas para comprender los contextos en los que los estudiantes interactúan. La lectura del contexto permite identificar y analizar elementos del entorno que deben fortalecerse, modificarse o transformarse para hacer posible una ciudadanía activa, crítica y responsable.

Las anteriores exigencias son fundamentales en un país como Colombia que en el mediano plazo está pensando en solucionar su conflicto político por la vía de la negociación, lo que exige a las comunidades, entre ellas a las educativas, un compromiso serio y responsable con la paz en sus territorios (López, 2016); al mismo tiempo demanda formar para la ciudadanía global, que implica formar ciudadanos del mundo, que se reconozcan como habitantes del planeta, responsables de su cuidado, sostenibilidad y de la construcción de solidaridad planetaria.

La investigación no es pues una alternativa para acercarse a mejores conocimientos, aunque ello se logra; ella debe aparecer como la herramienta fundamental para comprender los escenarios locales en que los estudiantes se desenvuelven y su relación con el contexto global, permitiendo a las comunidades educativas comprender que cada vez dependemos más del extenso e incomprensible mundo que ignoramos.

\section{Habilidades investigativas para la convivencia y la formación democrática}

En una encuesta realizada en la Institución Educativa Pedro Grau y Arola, de la ciudad de Quibdó, en el desarrollo de la investigación arriba mencionada, se pudo 
constatar que los estudiantes tienen una idea bastante precaria de la ciudadanía y de la democracia, y de esta en el ámbito escolar. Para ellos la democracia, y la democracia escolar no están sustentadas en la participación, en el ejercicio y control de lo público, sino en el desarrollo de actividades formales, a lo que se suma la idea tan impulsada por los medios de que son más importantes los candidatos y su imagen que sus programas.

En esta dirección es pertinente reconocer que la tarea fundamental de la educación en el campo de la democracia tiene que ver con formar en y para la dignidad y la libertad; para estimular la convivencia pacífica y la construcción de una cultura de paz entre las sociedades, tal como lo propone el Decreto 1038 de 2015 sobre la Cátedra de la Paz. La tarea de la formación ciudadana tiene que ser una labor pedagógica, política y jurídica, es decir, formativa, que permita actuar en contexto y que a partir de ella se procure el reconocimiento y ejercicio de los derechos de los individuos (Naciones Unidas, 1999).

Una propuesta pedagógica de educación en y para la formación ciudadana debe considerarse desde una perspectiva ética y política, vinculada con los problemas que enfrenta el entorno inmediato de los estudiantes como son la pobreza, la injusticia social, la violencia, la discriminación, el miedo y la corrupción (Toro, 2007). En igual dirección es fundamental reconocer a los docentes de las escuelas y colegios del país como intelectuales y como investigadores, lo cual se hace evidente, no porque ellos hagan "ciencia" sino, como bien lo proponen los Lineamientos curriculares en Ciencias Sociales (MEN, 2002), porque aprovechan con los estudiantes las estrategias y los métodos que les brinda el conocimiento científico; de allí que sea pertinente retomar la sugerencia que ha planteado la docente e investigadora Marina Camargo, de la Universidad de la Sabana: "Maestro investigador, ¿y por qué no?" (2006).

Es por ello que el presente artículo defiende la tesis de que una propuesta para trabajar el campo de la ciudadanía con los estudiantes debe hacer énfasis en la formación en el campo investigativo, donde al tiempo que estos se sensibilizan y forman para la democracia, accedan a competencias investigativas, lo que, de paso, les permite a las instituciones contar con un importante caudal de información sobre sus territorios, aprovechando mejor estos conocimientos para contextualizar sus saberes.

Con esta propuesta se pretende formar integralmente ciudadanos líderes, responsables de sus decisiones individuales y colectivas, críticas y reflexivas frente a la realidad y al acontecer social. Hombres y mujeres con voluntad para aprender y desaprender, capaces de emprender acciones para vivir con otros en medio de la diferencia, donde el pluralismo, la relación intergeneracional y la diversidad tengan cabida. 
Se reconoce así que lo importante de la formación ciudadana parte de considerar que quien conoce sus garantías y libertades está capacitado para demandar, reclamar, exigir y proponer; por ello a este tipo de sujetos es menos probable que se les atropelle en su integridad.

Se trata, pues, de sensibilizar a los estudiantes sobre lo que pasa en las comunidades donde ellos se desenvuelven. Una propuesta como estas procura no solo responder a la pregunta de cómo me entero mejor de un problema, sino de cómo me conmuevo con esa realidad que reflexiono. No solo se trata de analizar cómo me informo, sino cómo me transformo. De allí las exigencias, en el campo de las competencias actitudinales y volitivas.

La formación ciudadana supone educar desde y para unos determinados valores tales como la justicia, la cooperación, la solidaridad, el compromiso, la autonomía personal y colectiva, el respeto, etc., al mismo tiempo que se cuestionan y descalifican aquellos que aparecen como antivalores como son la discriminación, la intolerancia, la violencia, la indiferencia, la insolidaridad, el conformismo, etc.; como bien lo sugiere el investigador chileno, Humberto Maturana:

Educar se constituye en el proceso por el cual el niño, el joven o el adulto convive con otro y al convivir con el otro se transforma espontáneamente, de manera que su modo de vivir se hace progresivamente más congruente con el otro en el espacio de convivencia. Si el niño, joven o adulto no puede aceptarse y respetarse a sí mismo, no aceptará ni respetará al otro. Temerá, envidiará o despreciará al otro, pero no lo aceptará ni lo respetará y sin aceptación y respeto por el otro como un legítimo otro en la convivencia no hay fenómeno social (1992, p. 17).

La educación desde una perspectiva política busca formar un sujeto de derechos que actúe ética, moral y democráticamente en su contexto. Sin compromiso democrático de los ciudadanos y sin responsabilidad ética ni moral solo será posible un mundo de confrontación y guerras, de individuos insolidarios, de sujetos solitarios que solo piensan en lo que les conviene, un mundo en el que la gente hace lo que le da la gana y no lo que quiere, un mundo donde los medios masivos les imponen las conductas a los ciudadanos.

Para formar desde las instituciones educativas en el campo de la ciudadanía, la democracia y la investigación es de suma importancia el uso de estrategias como: (García y Ospina, 2015, p. 7)

- Talleres sobre ciudadanía y democracia.

- Construcción de diagnósticos participativos con enfoque de derechos.

- Consulta de diversas fuentes de información. 
- Construcción de historias de vida.

- Elaboración de ensayos, mapas conceptuales y cartografías sociales.

- Participación en eventos territoriales.

- Recorridos urbanos y regionales.

- Socialización de trabajos, exposiciones y conversatorios.

- Informes de investigación en diversos formatos (vídeos, exposición fotográfica, artículos de revista, pósters).

Estas estrategias superan lo que tradicionalmente se hace en las instituciones educativas de la básica y media cuando de investigar y de formar en el campo de la ciudadanía se trata.

Mientras que la formación en el campo de la investigación tradicional se sustenta en las consultas aisladas en enciclopedias e Internet, en la soledad de los estudiantes en los procesos de consulta e investigación en los que los resultados finales se circunscriben a la entrega de un trabajo, donde investigar aparece como un evento aislado y los trabajos no tienen resonancia social, las estrategias que se están proponiendo buscan un acercamiento permanente y sistemático a diversas fuentes de información (revistas, periódicos, audiovisuales, fuentes primarias, etc.), acompañamiento y capacitación permanente en métodos y técnicas de investigación social, socialización, sustentación y retroalimentación permanente de los avances de las investigaciones, monitorización y articulación de los procesos, acercamiento a fuentes, técnicas y sistematización. Propuestas como estas deben asumirse como estrategias transversales que atraviesan todo el currículo y el plan de área.

Una propuesta de estas calidades debe ser transversal, pues educar en este campo involucra diferentes ámbitos como son las dimensiones afectiva, intelectual, cognoscitiva, ética y de convivencia. Desde esta óptica la transversalidad no consiste en hacer malabares para "meter" los temas de las diversas asignaturas en una determinada propuesta; consiste en aprovechar mejor todos los espacios formativos de que se puede valer un proyecto pedagógico y la institución para mejorar su quehacer (Magendzo, 2003). La transversalidad no consiste en sumar disciplinas, sino en recuperar sus desarrollos en función de los problemas que se desea abordar.

\section{A manera de epílogo}

Si bien en el presente trabajo se está defendiendo la idea de que la investigación escolar es una excelente estrategia para promover la formación ciudadana de los 
estudiantes, la propuesta logra configurarse a partir de los hallazgos del trabajo de campo realizado en la Institución Educativa Pedro Grau y Arola.

Se encontró en la institución que existen serios problemas de convivencia entre los estudiantes que les impiden vivir a plenitud la ciudadanía, entendida como el ejercicio de sus derechos, problemas debidos a discriminación, convivencia en el interior de la institución, los cuales se resuelven de manera inmediata y tradicional: inmediata porque en la mayoría de los casos son los docentes quienes logran intervenir estos asuntos y es tradicional porque no se siguen protocolos o rutas previamente definidas, sino que se dialoga con los implicados, sin que se logre establecer un seguimiento que garantice la no repetición de actos violatorios de los derechos de los estudiantes.

Llama la atención que el $43 \%$ de los estudiantes haya sentido agresiones, discriminaciones o formas de exclusión en el interior de la institución, lo cual denota que los espacios educativos aún no son territorios donde la ciudadanía, el respeto por las diferencias y la tolerancia se hayan instalado con fuerza; de allí la precaria formación en el campo de la ciudadanía. Los espacios educativos que deberían ser por excelencia los territorios para el disenso no encuentran formas para que las diferencias sean ampliamente expresadas.

Respecto a la manera como se debería intervenir la comisión de las faltas, los estudiantes consideran que la institución debería hacerlo a partir de la aplicación de sanciones; lo que da cuenta del poco trabajo que en materia de convivencia, prevención de la violencia y de justicia escolar se ha hecho en la institución, que hasta el momento no cuenta con una rutas y protocolos definidos para afrontar estas situaciones.

Es bien diciente que la cultura escolar y las formas de entender la justicia escolar se hayan sustentado en buena medida en el castigo, y que las formas de justicia restaurativa, la negociación y el diálogo no aparezcan con fuerza; igualmente es importante que muchos estudiantes estén reclamando la necesidad de campañas encaminadas a promover la convivencia y el respeto por el otro.

Se evidenció que la Institución Educativa Pedro Grau y Arola, para 2015, no había definido los protocolos para mediar ante los diferentes conflictos, según lo indicado por la Ley 1620 de 2013 y el Decreto 1965 de 2013, sobre convivencia escolar y formación para el ejercicio de los derechos humanos, la educación para la sexualidad y la prevención y mitigación de la violencia escolar.

Así planteadas las cosas, y en la dirección que se construye el presente análisis, es posible sugerir que una escuela preocupada por la formación democrática y ciudadana de sus estudiantes a través del desarrollo de habilidades investigativas requiere, necesariamente, de maestros como lo sugería Henry 
Giroux (1997), que se asuman como intelectuales transformadores, no como reproductores de currículos prefabricados que los desconocen como sujetos cargados de saberes y experiencias, que han logrado construir una postura ética y política frente al quehacer de la educación. Es la idea de un docente como intelectual de la pedagogía, no como operador del saber.

A una propuesta como estas debe preocuparle e indignarle, en términos éticos, morales y políticos la exclusión social, la discriminación, las agresiones contra las personas; en una palabra, los actos violatorios de los derechos humanos; de igual manera es necesario defender el derecho a habitar en el mundo sin ser maltratados; entender que existen atentados y agresiones que no se pueden permitir. Es por eso que se concibe que el problema fundamental de la educación consiste en resolver el tipo de sujeto que se quiere formar el cual, a toda costa, debe ser un modelo que humanice a las personas.

Formar en democracia y ciudadanía debe asumirse como una necesidad existencial, pues con frecuencia se pide a la escuela que forme a unos sujetos de los que aún la sociedad en su conjunto carece, pues nadie nace demócrata, nadie nace conviviente, nadie nace honrado, nadie nace violento; todos esos son aprendizajes artificialmente construidos, y aunque de ello no se da cuenta la gente, toda la sociedad los paga o los disfruta.

La escuela tiene que reconocer que hoy emergen en los escenarios públicos nuevas identidades; de allí la necesidad de un proceso pedagógico para su visibilización e inclusión. Es necesario desde las instituciones educativas desarrollar actitudes de inclusión de las diferencias, de tal manera que las expresiones relacionadas con las etnias, el género, las edades, la orientación sexual, las víctimas de violencias y agresiones, etc., conocidos como poblaciones diversas, diferenciadas o vulnerables, sean plenamente respetadas. Es fundamental la promoción de comunidades incluyentes, democráticas y protectoras. No se trata de la apuesta por lógicas reproduccionistas del saber pedagógico, sino de fortalecer procesos donde las subjetividades sean reconocidas.

De una verdadera educación política y democrática deben resultar ciudadanos autónomos, sensibles con las problemáticas de sus comunidades y tejidos sociales fortalecidos; situación que no ocurre cuando se habla de la educación vertical, pues una sociedad autoritaria produce una escuela autoritaria. Una propuesta como estas debe asumir la tarea de la formación en el campo de la democracia como experiencia humanizante y praxis pedagógica; de allí que se plantee la necesidad de un currículo que ponga en diálogo los distintos saberes, desde un enfoque de derechos y con una perspectiva crítica y diferencial.

En medio de las dificultades que hoy presenta el sistema educativo colombiano, muy especialmente en lo que respecta a la convivencia escolar, se 
reconoce que en la actualidad las instituciones han experimentado profundos cambios en su manera de asumir la labor formativa, lo cual ha sido dinamizado en buena parte por el arribo de maestros más capacitados, más jóvenes y de mayor apertura a los estudiantes; ellos han brindado nuevas dinámicas de identidad y pueden ser interesantes como puntos de apoyo para diseñar procesos de sensibilización en la convivencia ciudadana, en el respeto por los derechos de los demás, en la solidaridad social; pero estos elementos serían insuficientes si el Estado no hace presencia social en las comunidades más marginadas y excluidas de la sociedad.

Es por lo anterior que es muy difícil que se pida a las instituciones educativas que asuman de manera pacífica y mediante el diálogo civilizado los conflictos, en una sociedad y en un país que no ha logrado asumirlos. Pese a ello son numerosas las instituciones que vienen dando muestras de estar dispuestas a correr el reto de aportar su granito de arena en la generación de una sociedad plural, para construir en sus espacios cotidianos unas relaciones interpersonales en las que todos tengan cabida.

\section{Referentes bibliográficos}

Agudelo, E. (2006). El nuevo enfoque curricular para la enseñanza de las ciencias sociales en la educación básica y media. Nueva ciencias sociales. Propuesta curricular integrada (libro guía para el docente). Bogotá: Grupo Editorial Educar.

Arteta, A. (2008). Aprender democracia, ¿por qué? El saber del ciudadano: las nociones capitales de la democracia. Madrid: Alianza Editorial.

Ausbel, D. (2002). Adquisición y retención del conocimiento: una perspectiva cognitiva. Madrid: Paidós.

Camargo, M. (2005, enero). Maestro investigador ¿y por qué no? Bogotá: Universidad Pedagógica Nacional, pp. 24-31.

Camargo, M. (2006). Política educativa, equidad y formación docente. Bogotá: Universidad Pedagógica Nacional.

Castells, M. (2002). La era de la información. La sociedad red. Bogotá: Planeta.

Colciencias-Ondas. (2007). Xua, Teo y sus amigos en la Onda de la investigación. Guía de la investigación y de la innovación. Bogotá: Fundación Fes-Colciencias.

García, D. y Ospina, E. (2015). Orientaciones básicas para la implementación de la cátedra de la Paz en las instituciones educativas de Medellín, 2015. Medellín: Secretaría de Educación.

Gil, M. (2011, noviembre). La construcción de la memoria histórica de las víctimas de la violencia. Desde la Región, Medellín: Corporación Región, N. 554, pp. 37-48.

Giroux, H. (1997). Los profesores como intelectuales. Hacia una pedagogía crítica del aprendizaje. Madrid: Paidós.

Jares, X. (1997). El lugar del conflicto en la organización escolar. Revista Iberoamericana de Educación, N. ${ }^{\circ} 15$, septiembre-diciembre, pp. 3-7. 
López, C. (2016). iAdiós a las Farc! ¿Y ahora qué? Bogotá: Debate.

Maguendzo, A. (1999). Currículum, educación para la democracia en la modernidad. Bogotá: Instituto para el Desarrollo de la Democracia Luis Carlos Galán Sarmiento.

Magendzo, A. (2003). Transversalidad y currículum. Bogotá: Magisterio.

Maturana, H. (1992). Emociones y lenguaje en educación y política. Santiago: Hachette.

Mclaren, P. (2005). La vida en las escuelas. Una introducción a la pedagogía crítica en los fundamentos de la educación. México: Siglo XXI.

Ministerio de Educación Nacional. (2002). Lineamientos curriculares en Ciencias Sociales. Bogotá.

Ministerio de Educación Nacional. (2015). Creación de la cátedra de la Paz. Bogotá.

Oficina en Colombia del Alto Comisionado de Las Naciones Unidas para los Derechos Humanos-Unión Europea. (2003). Compilación de instrumentos internacionales: Derecho Internacional de los derechos Humanos, Derecho Internacional Humanitario y Derecho penal Internacional. Bogotá.

Ospina, E. (2000). Formación ciudadana. Tesis de grado (Maestría en Sociología). Medellín: Universidad de Antioquia.

Ospina, E. (2015). Pedagogías de la memoria: el papel de la escuela en el posconflicto. Ponencia presentada en la Primera Bienal Internacional de Educación y Cultura de Paz. San Agustín, Huila.

Pérez, G. (1994). Investigación cualitativa. Retos e interrogantes. Madrid: La Muralla.

Tibbitts, F. (2007, mayo). Nuevos modelos de educación en derechos humanos. San José de Costa Rica. Vol. 15, N. $^{\circ} 7$.

Toro, J. (2007). Educación para la democracia. Bogotá. Fundación Social.

Vásquez, A. (2008). Zygmunt Bauman: Modernidad líquida y fragilidad humana. Observaciones filosóficas, N. ${ }^{\circ}$ 6. Bogotá: Universidad de los Andes, pp. 1-8. 
